\title{
Passive fluxgate control of structural transformations in structural steels during thermal cycling
}

\author{
Alexander Scherbakov ${ }^{1 *}$, Daria Monastyreva ${ }^{2}$, and Vitaly Smirnov ${ }^{3}$ \\ ${ }^{1}$ Saint Petersburg State University of Architecture and Civil Engineering, St. Petersburg, Russia \\ ${ }^{2}$ Peter the Great St. Petersburg Polytechnic University, St. Petersburg, Russia \\ ${ }^{3}$ Moscow Aviation Institute, Volokolamskoe highway, 4, Moscow, 125993, Russia
}

\begin{abstract}
Thermocycler processing (TCO) of metals and alloys belongs to one of the most effective ways to obtain the structure of structural steels with a given degree of dispersion. However, the development of thermocycler processing modes in each case is individual in nature and cannot be mechanically transferred from one material to another. Therefore, it is necessary to consider the structural changes in metals during TCO using examples of steels belonging to different groups so that from the whole variety of processes, dissolution and precipitation of phases, stresses and strains, only those that allow you to obtain a given optimal fine-grained structure are selected. Based on the obtained data, we plotted the dependence of the magnetic field strength $\mathrm{Hp}$ on the number of cycles during thermal cycling. A comparative analysis of the obtained results is made and conclusions are drawn on the relationship between the magnetic parameter $\mathrm{Hp}$ and structural changes in steels during TCO.
\end{abstract}

\section{Introduction}

Thermocyclic treatment (TCO) of metals and alloys belongs to one of the most effective methods for obtaining the structure of structural steels with a given degree of dispersion [1$3]$. Its basis is the constant accumulation, from cycle to cycle of heating and cooling, of positive changes in the structure of metals. One of the important features of the metal heating - cooling cycle is its intensity, the absence or presence of small exposures at extreme temperatures, and also a sufficient range of temperature changes [4]. In this case, additional sources of influence on the structure appear, the main of them: phase transformations in the metal, temperature gradients, thermal (bulk) and interfacial stresses. It should be emphasized that with TCO, it becomes possible to obtain microstructures with a given dispersion characteristic of the supplied factory steel and structural heterogeneity of welded joints $[5,6]$. In this case, it is possible to obtain such a fine-grained structure, which is unattainable with conventional types of heat treatment $[7,8]$. This, in turn, allows the use of thermocyclic treatment to enhance the hazardous zones of stress concentration $(\mathrm{KH})$ in the elements of welded metal structures (MK) and welded joints with structural

\footnotetext{
*Corresponding author: shurbakov.aleksandr@yandex.ru
} 
heterogeneity due to the formation of a finer-grained structure with increased strength properties in them.

In [9], a comparative analysis of the mechanical properties obtained by quenching with subsequent tempering after tempering was carried out for steel 30KhGSA. The data obtained allowed us to conclude that it is advisable to use a TCOt to improve not only plastic but also strength characteristics.

Obtaining coarse-grained structures in structural steels is carried out by hightemperature annealing, while obtaining a fine-grained structure with varying degrees of dispersion is a serious problem [10]. The analysis showed that the most effective way to obtain a microstructure with a given degree of dispersion is thermocyclic treatment, which consists of periodically repeated heating and cooling in regimes that take into account the internal structure of the material: the difference in the thermophysical characteristics of the phases, the volume effect of phase transformations, etc. Such an approach makes it possible in a fairly short time to form the optimal fine-grained structure in materials. However, the development of thermocyclic processing modes in each case is individual in nature and cannot be mechanically transferred from one material to another. Therefore, it is necessary to consider the structural changes in metals during TCO using examples of steels belonging to different groups so that from the whole variety of processes, dissolution and precipitation of phases, stresses and strains, only those that allow you to obtain a given optimal finegrained structure are selected.

Such a process of formation of a fine-grained structure in the process of TCO is controlled by the passive flux-gate method [11-13]. Fluxgate control method is a magnetic non-destructive testing method based on registration of scattering magnetic fields of a test object by fluxgate transducers. A flux-probe flaw detector is a magnetic flaw detector that uses flux-gate transducers as sensitive elements. Fluxgate transducer - ferromodulation transducer with permalloy cores. The defect scattering magnetic field is a local magnetic field arising in the defect zone due to the magnetic polarization of its boundaries. The sensitivity of the fluxgate control method is determined by the magnetic characteristics of the material of the controlled product, its shape and size, the control method and type of magnetization, the sensitivity of the transducer and electronic equipment used, and also the defect's magnetic field. It should be noted that the method allows you to control products of such sizes and shapes in which the ratio of their length to the largest size in the transverse direction and their magnetic properties allow magnetization to a degree sufficient to create a deflecting magnetic field detected by the transducer, which reduces the possibility method.

The influence of the chemical composition and structural state of steels is studied by changing the magnetic parameter $\mathrm{Hp}$, which characterizes the magnetic field strength.

The purpose of this article is to establish the relationship between the magnetic parameter Hp and structural changes in steels during TCO.

As part of the goal, tasks such as:

1. To test samples of low-carbon steel 08ps and low-alloy steel 10HSND in various conditions: in the state of factory delivery; supply + annealing at $900^{\circ} \mathrm{C}$; supply + cold rolling at a degree of deformation $\varepsilon=50 \%$.

2. Based on the obtained data, construct graphs of the dependence of the magnetic field strength $\mathrm{Hp}$ on the number of cycles during thermal cycling.

3. To carry out a comparative analysis of the results and formulate conclusions about the relationship between the magnetic parameter $\mathrm{Hp}$ and structural changes in steels during TCO. 


\section{Methods}

For experimental research, samples were cut from 08ps low-carbon steel and 10KhSND low-alloy steel, which were subjected to TCO in various conditions: in the state of factory delivery; supply + annealing at $900^{\circ} \mathrm{C}$; supply + cold rolling at a degree of deformation $\varepsilon$ $=50 \%$. The composition of the steels is presented in table 1 .

Structural changes in the metal during the TCO were monitored by the passive flux-gate method using microstructural analysis and solidometry. In this case, an IKNM-2FP device was used with a two-channel flux-gate transducer mounted perpendicular to the sample surface. In the middle part of the sample, the values of the magnetic parameter of the magnetic field strength $\mathrm{Hp}$ were recorded along the axial line in three control zones separated by $30 \mathrm{~mm}$ from each other. Measurements of Hp were carried out both before the first TCO cycle and at the end of each heating-cooling cycle. When plotting the dependences of the $\mathrm{Hp}$ values on the number of cycles, the averaged $\mathrm{Hp}$ value of the indicated control zones was adopted. The number of cycles was as follows: 1, 2, 3, 4, 5, 7, and 10 .

Before thermocyclic treatment, the test samples in the delivery state underwent a 30minute annealing at $900^{\circ} \mathrm{C}$, due to which an equilibrium structure was formed in them.

Table 1. The chemical composition of low-carbon steel 08ps and low-alloy steel 10HSND

\begin{tabular}{|c|c|c|c|c|c|c|c|c|c|}
\hline \multirow{2}{*}{ Steel } & \multicolumn{10}{|c|}{ Chemical composition, \% } \\
\cline { 2 - 11 } & $\mathbf{C}$ & $\mathbf{M n}$ & $\mathbf{S i}$ & $\mathbf{A l}$ & $\mathbf{P}$ & $\mathbf{S}$ & $\mathbf{C r}$ & $\mathbf{N i}$ & $\mathbf{C u}$ \\
\hline 08ps & 0,06 & 0,27 & 0,05 & 0,0011 & 0,019 & 0,022 & - & - & - \\
\hline 10HSND & 0,12 & 0,57 & 0,92 & - & 0,032 & 0,036 & 0,63 & 0,87 & 0,48 \\
\hline
\end{tabular}

To conduct research, plates $30 \mathrm{~mm}$ wide, $150 \mathrm{~mm}$ long, and $2 \mathrm{~mm}$ thick were cut from sheets of 08ps low-carbon steel and low-alloy 10KHSND across the rolling line (Fig. 1). Samples for mechanical tests in accordance with GOST 1497-84 were made from the plates in the delivery and pretreated state. Metals Tensile test methods..

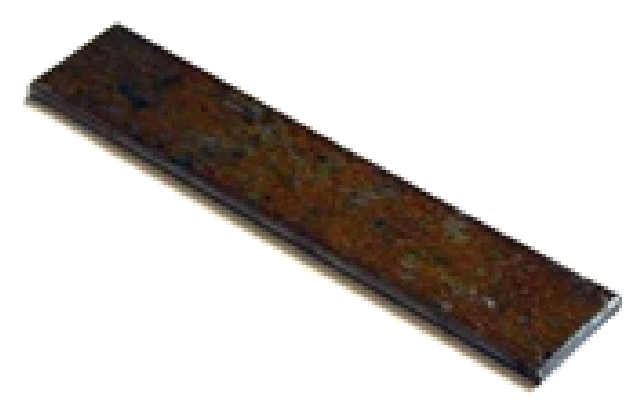

Fig.1. The bulk of the samples used for experimental studies

To assess the stress-strain state of welded load-bearing metal structures of SM made of ferromagnetic materials, a passive flux-gate control method was developed by measuring the magnitude of the magnetic field of the scattering by an IKNM-2FP device with a twochannel flux-gate converter (Fig. 2). The implementation of the methodology included two stages: preparing the device for operation and conducting non-destructive magnetic control 
of the metal. During the control, the places of abnormal changes in Hp were recorded and the degree of danger of the proposed zones of SC was assessed. A feature of the developed technique was that it allowed continuous and selective scanning of the surface of the base metal and welded joints, including in hard-to-reach places and without preliminary surface preparation and removal of a non-magnetic protective coating with a thickness of less than $3 \mathrm{~mm}$.

The applied device ICNM 2-FP consists of a measuring unit with batteries, a flux gate transducer and a connecting cable. The device is small-sized, has autonomous power, is easy to operate and configure, allows measurements to be made in hard-to-reach places. The principle of the device's operation is based on recording the intensity of the scattering magnetic field $\mathrm{Hp}$ on the controlled surface of the object when it is magnetized in a weak magnetic field of the Earth (Rayleigh region) and revealing the anomalous behavior of the scattering magnetic field in zones of stress concentration. IKNM-2FP has digital and sound indication of the measured data with the possibility of their registration in the memory unit (recording up to 1000 measurements).

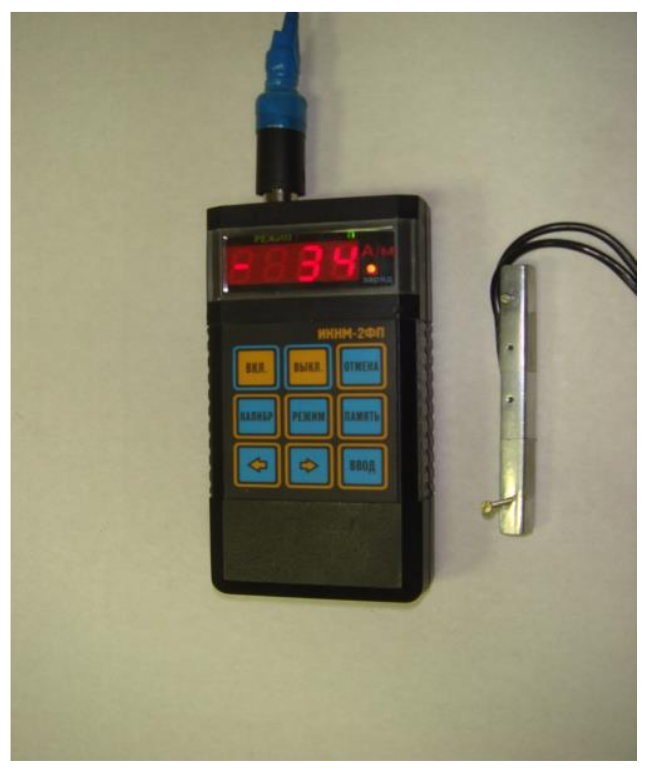

Fig. 2. Appearance of the IKNM-2FP device with a two-channel flux-gate converter

The sensor of the device has two flux gates located on the same axis with a varying base distance between them. The device makes it possible to work in the mode of a polemer or gradiometer (with a detuning from an external magnetic field). Technical characteristics of the device are given in table. 2 .

Table 2. Technical characteristics of the device IKNM-2FP

\begin{tabular}{|c|c|}
\hline Characteristic & Indicators \\
\hline Range of measurement of the value of $\mathrm{Hp}, \mathrm{A} / \mathrm{m}$ & \pm 1999 \\
\hline The main relative measurement error, $\%$ & 5 \\
\hline Additional absolute measurement error, A / m & 5 \\
\hline The number of measurement channels $\mathrm{Hp}$ & 2 \\
\hline
\end{tabular}




\begin{tabular}{|c|c|}
\hline Characteristic & Indicators \\
\hline $\begin{array}{c}\text { Time of one measurement, } \mu \mathrm{s} \\
\text { The time of continuous operation of the device from two } \\
\text { batteries }\end{array}$ & 10 \\
\hline Rated voltage, $\mathrm{V}$ & 2,4 \\
\hline Operating temperature range, ${ }^{\circ} \mathrm{C}$ & от -20 до +60 \\
\hline Relative humidity range, $\%$ & $45-80$ \\
\hline $\begin{array}{c}\text { Overall dimensions, } \mathrm{mm}: \\
\text { instrument } \\
\text { sensors }\end{array}$ & $120 \times 60 \times 25$ \\
cable length "sensor-instrument" & $5 \times 5 \times 30$ \\
\hline Weight with batteries, $\mathrm{kg}$ & 0,25 \\
\hline
\end{tabular}

In preparation for control, it is necessary:

1. connect the fluxgate transducer to the measuring unit;

2. turn on the device by pressing the "ON" key. At the same time, the digital indicators for measuring the field, the indicator of the operating mode "P" or "G" (pole meter or gradiometer, respectively), as well as an indicator of the status of the battery batteries should light up on the screen of the device;

3. keep the device in the on state ("warming up") for 15 minutes;

4. calibrate the instrument before taking measurements.

5. The procedure for calibrating the device is as follows:

6. set the indicator of the operation mode of the device to the state "P" - polemer;

7. press the "CALIBER" key, while the device goes into calibration mode;

8. set the required calibration magnetic field (40 A / m) corresponding to the Earth's magnetic field. At the same time, information " $\mathrm{H} 1 \uparrow$ " is induced on the screen;

9. install the device sensor with probe 1 up (the "+" field) and press the "ENTER" key. Information "H1 $\downarrow$ " is induced on the screen;

10. install the probe with probe 1 down (field "-") and press the "ENTER" key. Calibration is over;

11. if necessary, exit the calibration mode by pressing the "CANCEL" key.

When measuring, a fluxgate transducer is placed strictly perpendicular to the surface of the metal being monitored. Then, by moving the sensor over the surface of the sample, the change in the values of the magnetic field scattering $\mathrm{Hp}$ on the screen of the device is controlled. Places of sharp changes in Hp are noted, thus revealing the alleged zones of SC.

When conducting magnetic studies, in order to obtain reliable experimental results in some of the samples, artificial QW zones were created in the central part due to lateral radius grooves, in which magnetic field strengths Hp were measured during stepwise cyclic elastoplastic loading.

To ensure the stability of the obtained experimental data during shutdowns, in all cases it was envisaged to measure Hp at regular intervals.

The process of formation of a fine-grained structure in the process of TCO was controlled by the passive flux-gate method and the method of microstructural analysis. The Hp values were measured by a two-channel flux-gate transducer mounted perpendicular to the surface of the sample. On each sample in its middle part along the center line, Hp values were recorded in four control zones spaced $30 \mathrm{~mm}$ apart. The values of Hp were measured both before the first TCO cycle and at the end of each heating-cooling cycle. To plot the dependences of the Hp values on the number of cycles, the averaged Hp value of the indicated control zones was adopted. 
The initial samples of 08ps and 10KhSND steels in the delivery state with a thickness of $2 \mathrm{~mm}$ were subjected to fractional rolling to a degree of deformation of $8,15,22,30,40$, and $50 \%$. The magnetic field scattering on the surface of the samples during their deformation was measured along the center line at three points spaced $30 \mathrm{~mm}$ apart.

\section{Results and Discussion}

In fig. Figure 3 shows the dependence of the magnetic field strength of the scattering Hp on the number of cycles during the thermal treatment of steels 08ps and 10KhSND in various initial states. It can be seen that the largest changes in the Hp parameter for all the studied steels occur during the first two to three cycles of TCO. In this case, the magnetic background of the samples is erased, as evidenced by a change in the positive sign of Hp to negative and the convergence of the values of $\mathrm{Hp}$. An increase in the number of cycles stabilizes the magnetic field strength $\mathrm{Hp}$, which is noticeable in a decrease in the spread in the values of the parameter Hp. An increase in the number of cycles from 5 to 7 and 10 has practically no effect on the values of the magnetic parameter Hp.

The TCO of the samples in the delivery state leads to a sharp change in the values of the magnetic field scattering after the first cycle (Fig. 4). Subsequent cycles of thermal cycling do not change the sign of negative values of $\mathrm{Hp}$.

The performed annealing before the TCO shifts the initial Hp values to the region of negative values Fig. 5a), however, no further significant changes in the Hp parameter are observed. Cold plastic deformation has a significant effect on Hp during the first three cycles (Fig. 5, b), then this effect decreases, which indicates a significant convergence of the microstructure of steels with the number of cycles exceeding three. Therefore, such a dependence of $\mathrm{Hp}$ on the number of TCO cycles has a positive value, since it allows one to judge the completeness of the process of structure refinement under passive flux-probe control, where plastic deformation to one degree or another has passed during operation.

It follows from the above that, regardless of the grade of structural steel, after the fifth heating and cooling cycle, the difference between the maximum and minimum Hp values of the studied steels (for different initial structural states) is almost the same and amounts to 4 A / $\mathrm{m}$, which indicates a close final structural state of the data steels after TCO. An increase in the number of cycles to 10 practically preserves the spread in the values of the parameter Hp.

The chemical composition of steels has a significant effect on the change in the intensity of the scattering magnetic field $\mathrm{Hp}$, the parameter Hp undergoes the largest changes in 08ps low-carbon steel and the smallest on 10KHSND low-alloy steel (see Fig. 3).

The presence of different similar steel structures also has a significant effect on the change in the parameter Hp from the number of TCO cycles.

Thus, samples with a coarse-grained and more equilibrium structure (supply + annealing at $900^{\circ} \mathrm{C}$ ) compared with finer-grained samples in the delivery state (see Fig. 5, a and Fig. 4, respectively) have smaller changes in the Hp parameter from the first cycle . Samples that underwent preliminary cold plastic deformation correspond to large fluctuations in the Hp values, especially after the second and third cycles of TCO (see Fig. $5, \mathrm{~b})$. 
a)

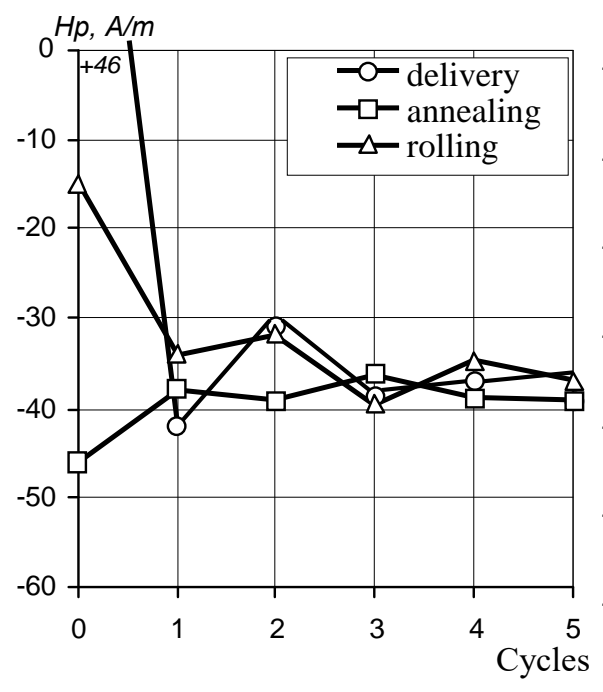

b)

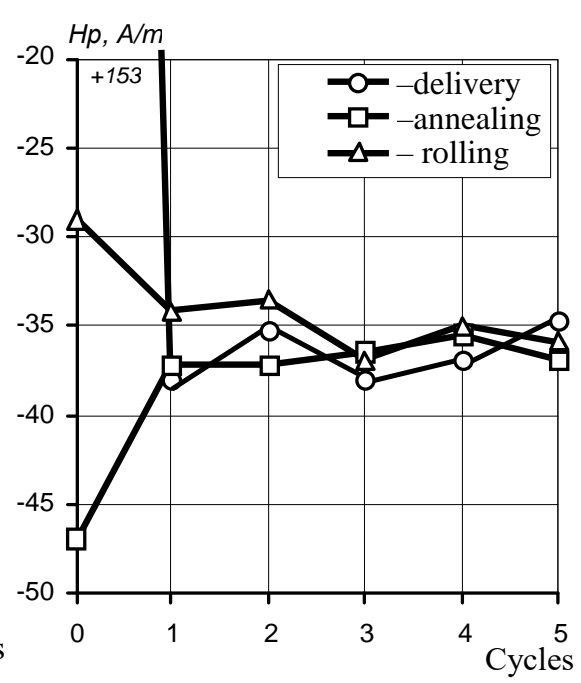

Fig. 3. Dependence of the magnetic field strength $\mathrm{Hp}$ on the number of cycles during thermal cycling of steels 08ps (a) and 10KHSND (b) in various initial structural states

It must be emphasized that a change in the $\mathrm{Hp}$ values depending on the chemical composition of the steels, the initial microstructure, and the number of TCO cycles is closely related to the structural changes of the metal that occur during the TCO process. Typical microstructures of steels after TCO are shown in Fig. 4-9. A metallographic analysis showed that, regardless of the initial structure of the samples, a fine-grained structure is observed in all steels after the third TCO cycle. A subsequent increase in the number of cycles grinds the structure of steels less significantly, which is in good agreement with the data on the change in the Hp parameter - in subsequent cycles, a decrease in the amplitude of fluctuations in the Hp values is noted.

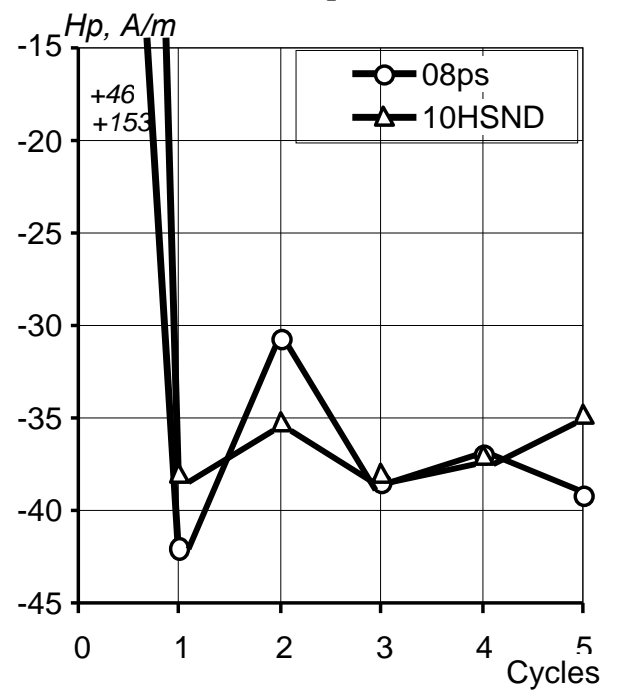

Fig. 4. The dependence of the magnetic field strength $\mathrm{Hp}$ on the number of cycles during thermal cycling of 08ps and 10KhSND steels 
a)

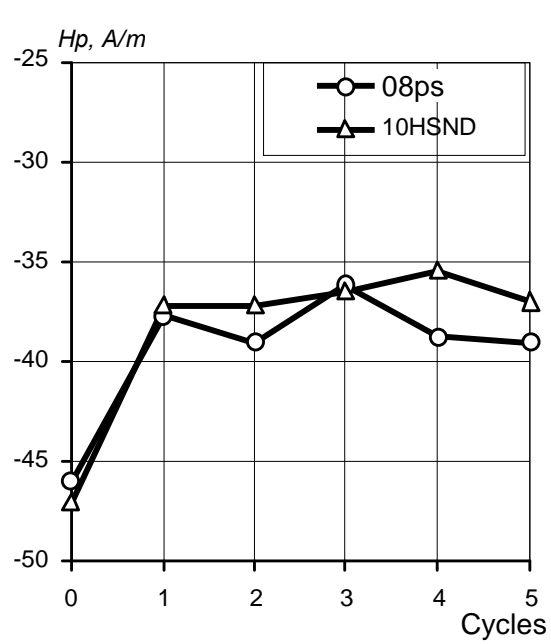

b)

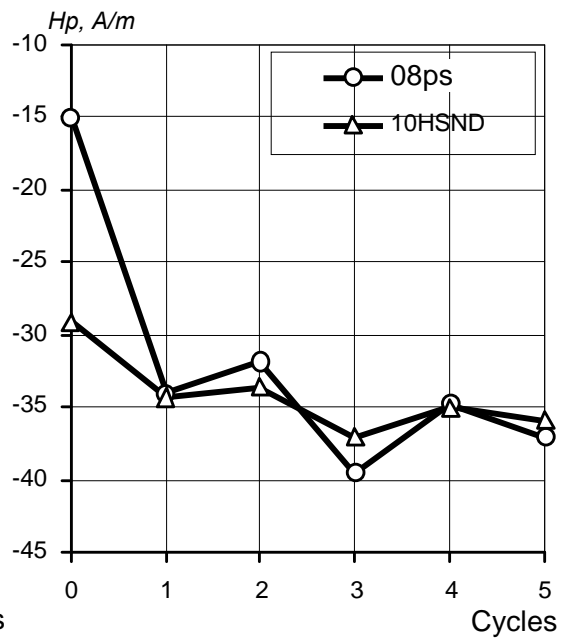

Fig. 5. Dependence of the scattering magnetic field $\mathrm{Hp}$ on the number of cycles during thermal cycling of 08ps and 10KhSND steels after preliminary annealing at $900{ }^{\circ} \mathrm{C}$ (a) and cold plastic deformation by $\varepsilon=50 \%(b)$

It should be noted that in 10KHSND steel, after 5-fold thermal cycling, a finer-grained structure is formed in comparison with 08ps low-carbon steel. In addition, in alloy steel, to obtain a fine-grained structure without noticeable different grain sizes, a greater number of TCO cycles is required than for carbon.

The difference between alloyed steels is that the thermodynamic activity of carbon in them is lower than in carbon steels. Therefore, the slowing down of the diffusion processes of cementite dissolution and its release from austenite leads to a shift of the C-shaped curve of isothermal decomposition of austenite to the right, making the supercooled austenite more stable. In addition, the previously described methods for carbon steel steels can be applied without a significant change in technology to pearlite alloy steels, when the total content of alloying elements does not exceed 5\%. TCO intensifies phase and structural transformations in steels, while the formation and propagation of new dislocations occurs, and with TCO of iron, an increase in the density of dislocations begins immediately after the start of processing. In this case, with an increase in the number of cycles (thermal cycling time), the dislocation density increases. Thus, the effectiveness of the TCO will be determined by the degree of generation of defects and, primarily, dislocations.

TCO stimulates a change in the microstructure, substructure, and dislocation structure of the metal, and the main structural change in steel is a significant refinement of grain size, regardless of whether it is perlite, ferrite, martensite, or other other structure. In TCO, grain refinement, as a rule, is accompanied by a decrease in the heterogeneity and a more uniform distribution of chemical elements.

The main reason causing such changes in the structure is a significant intensification of diffusion processes in steels due to the increased influence of thermophysical factors. In $\mathrm{TCO}$, the dislocations present in the metal begin to move, and new dislocations are formed and multiply. With the thermal stability of iron, zirconium, tungsten, as well as with lowtemperature thermal cycling of molybdenum, an increase in the density of dislocations begins immediately after the start of thermal cycling. 
a)

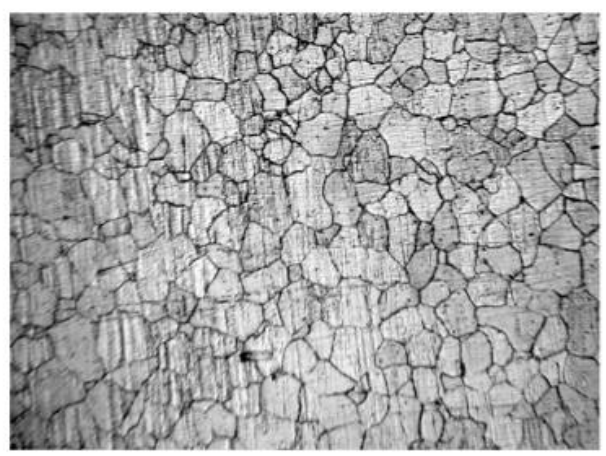

v)

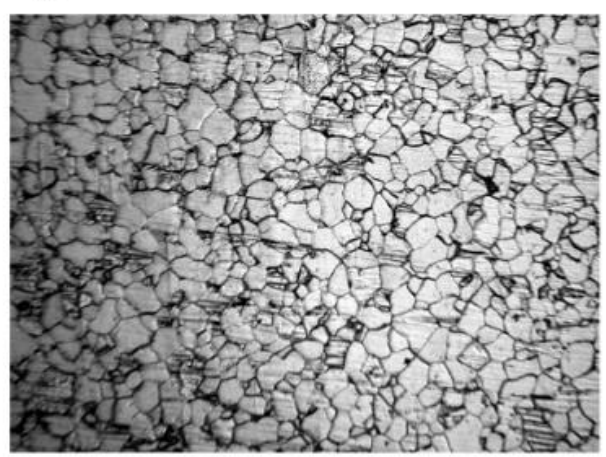

d)

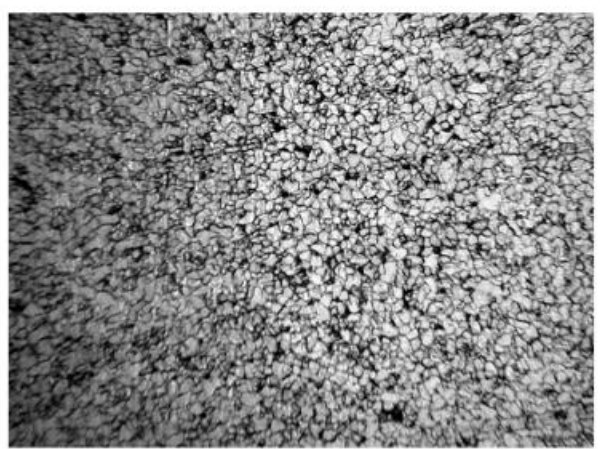

b)

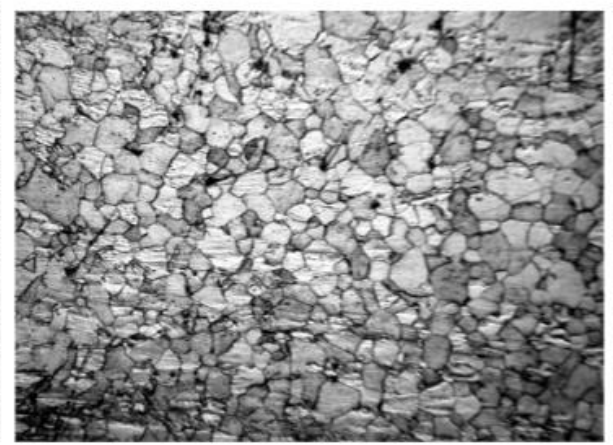

g)

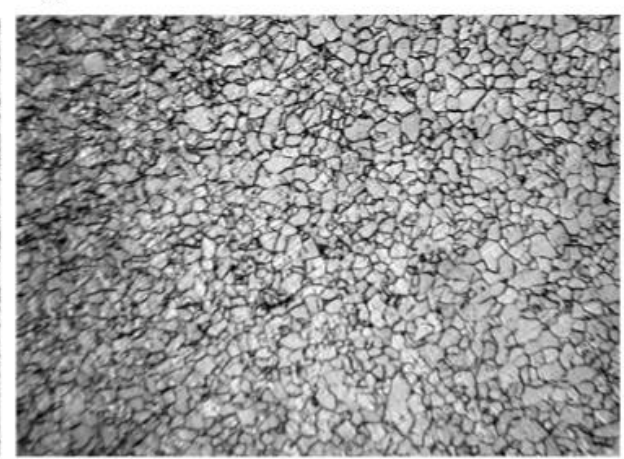

e)

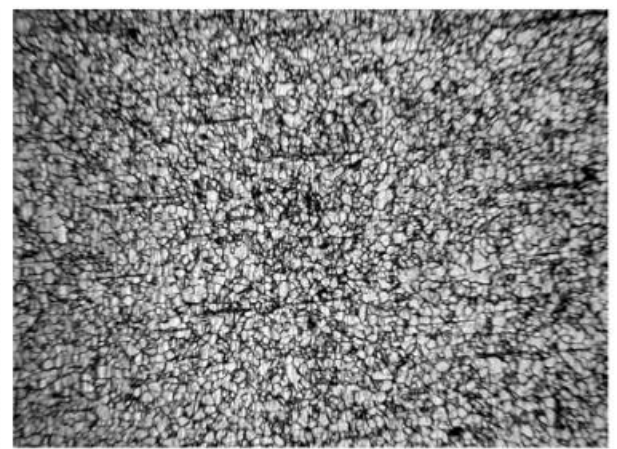

Fig. 6. Change in the structure of $08 \mathrm{ps}$ steel during thermocyclic processing, $\times 650$ : a - delivery state + annealing at $900^{\circ} \mathrm{C} ; \mathrm{b}-\mathrm{e}$ - after $1-5$ cycles, respectively 
a)

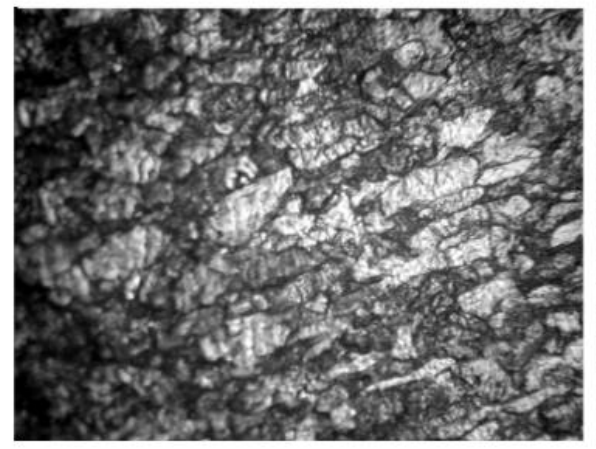

v)

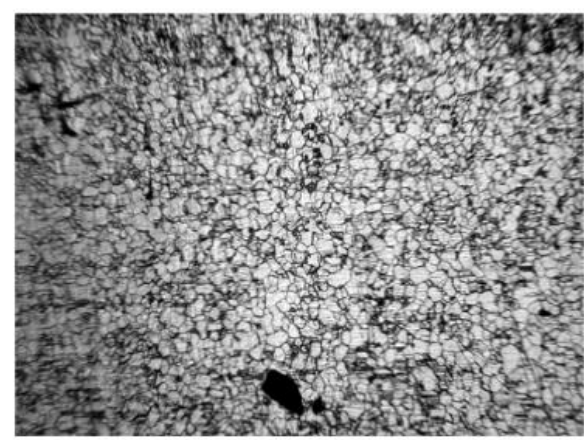

d)

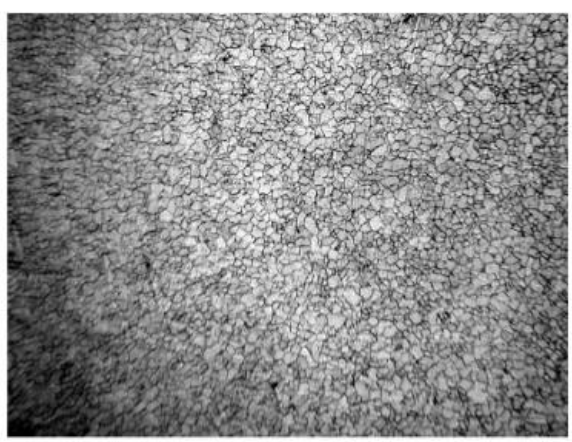

b)

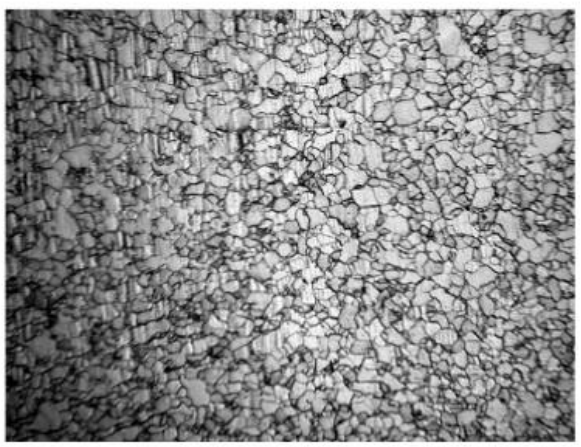

g)

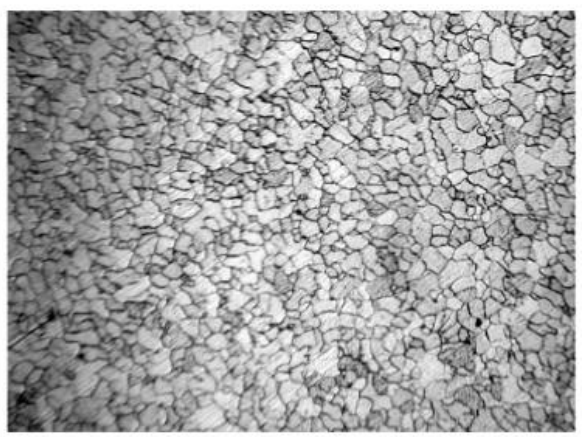

e)

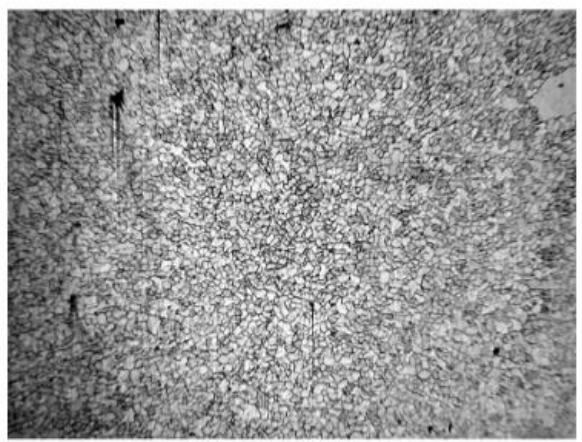

Fig. 7. Change in the structure of 08ps steel during thermocyclic processing, $\times 650$ : a - delivery + rolling condition at $\varepsilon=50 \% ; \mathrm{b}-\mathrm{e}$ - after $1-5$ cycles, respectively 
a)

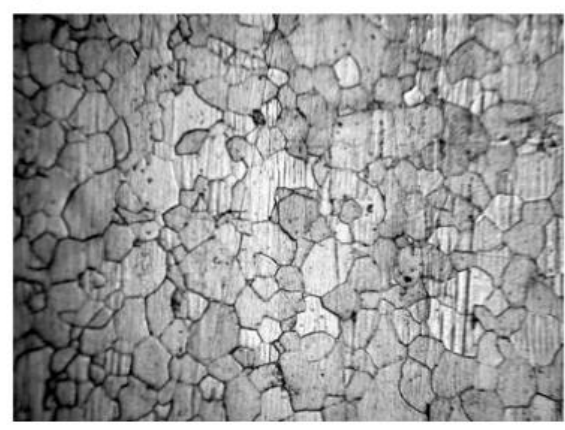

v)

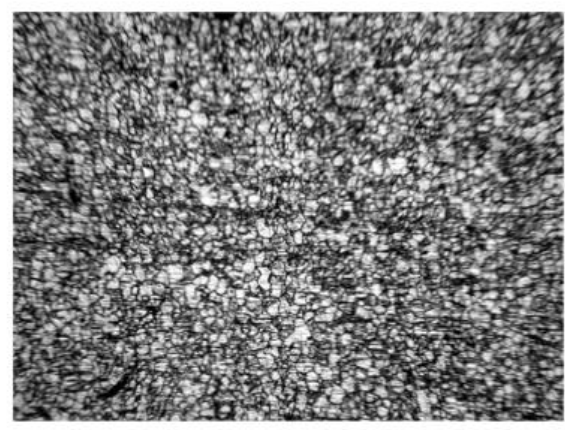

d)

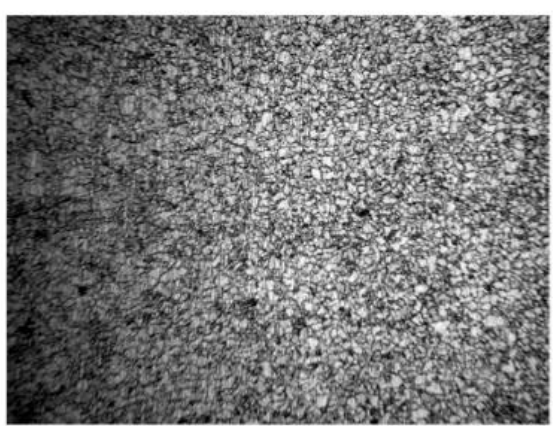

b)

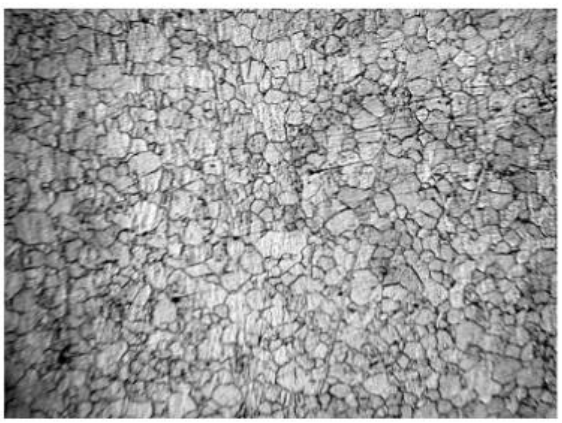

g)

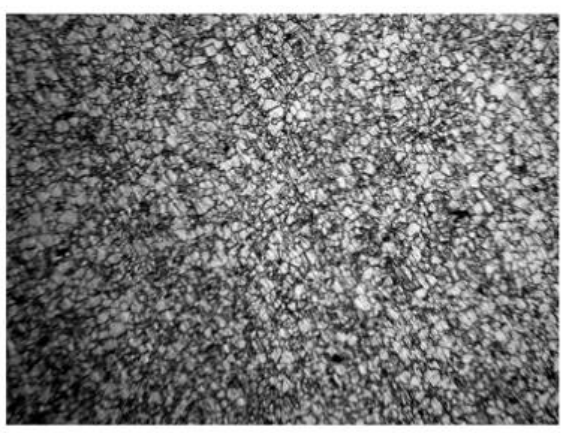

e)

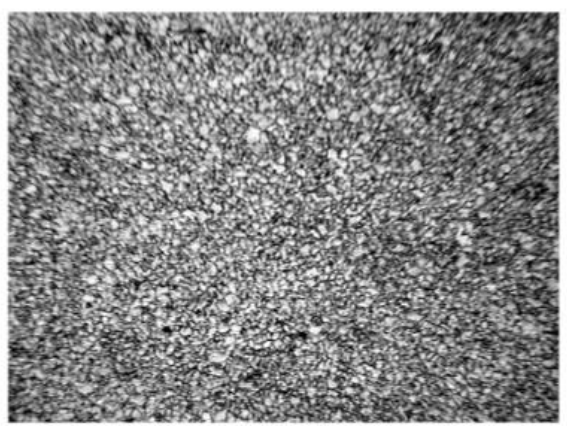

Fig. 8. Change in the structure of steel 10HSND during thermocyclic treatment, $\times 650$ : a - delivery state + annealing at $900^{\circ} \mathrm{C} ; \mathrm{b}-\mathrm{e}-$ after $1-5$ cycles, respectively 
a)

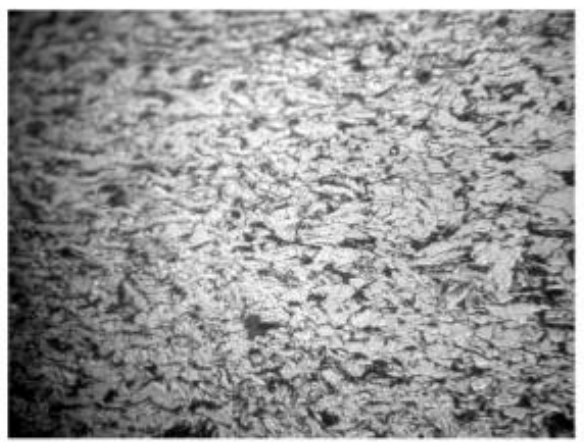

v)

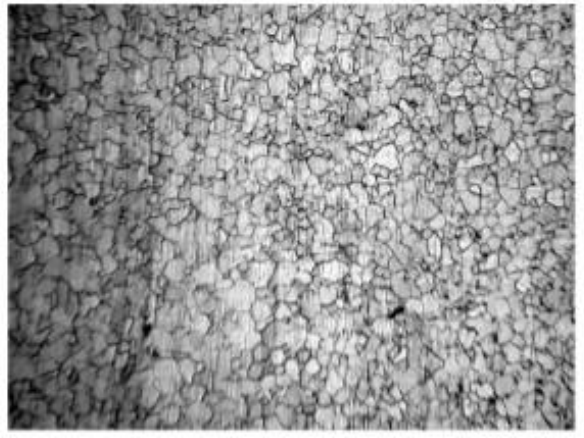

d)

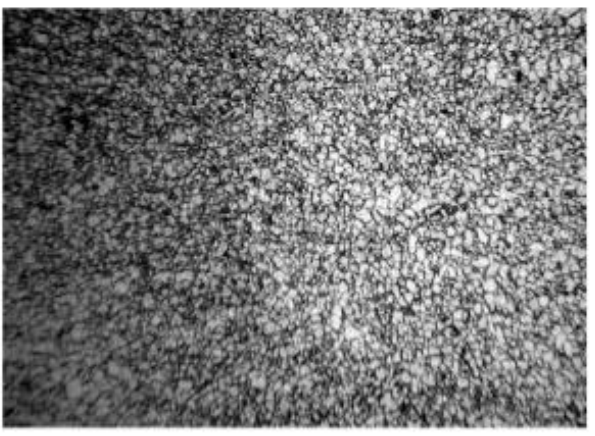

b)

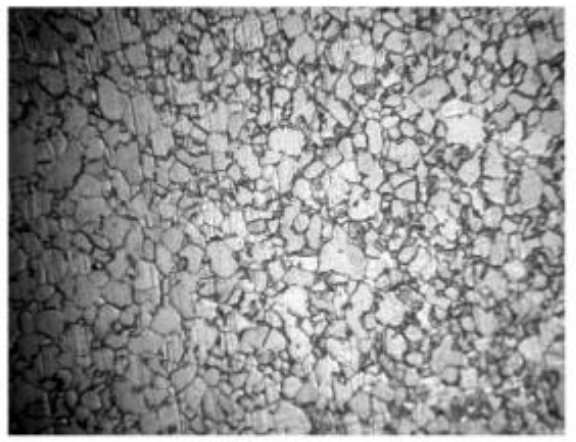

g)

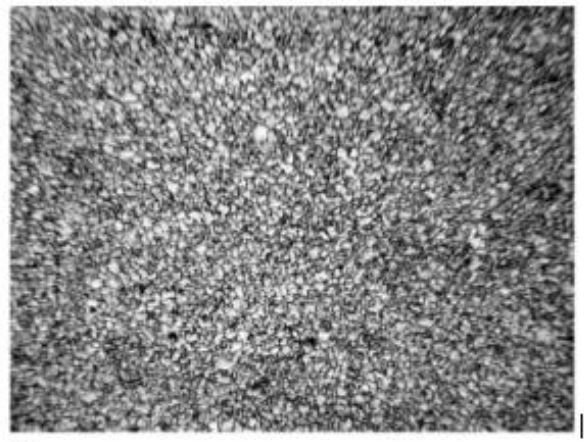

e)

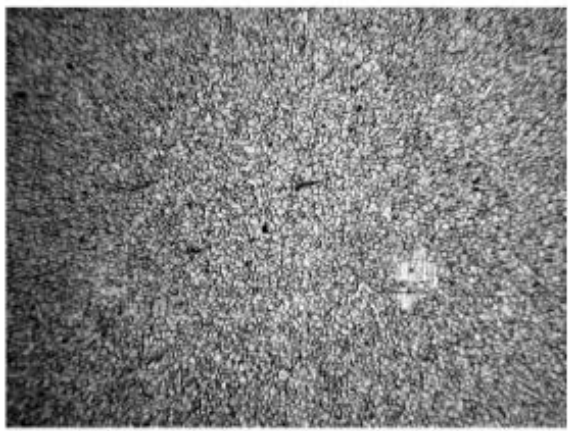

Fig. 9. Change in the structure of steel 10HSND during thermocyclic treatment, $\times 650: a-$ delivery + rolling condition at $\varepsilon=50 \% ; \mathrm{b}-\mathrm{e}-$ after $1-5$ cycles, respectively

An increase in the density of dislocations and the formation of a polygonal substructure after TCO of pure iron and low-carbon steel suggest that polymorphic transformations, leading to phase hardening due to the difference in specific volumes and elastic moduli of the formed phases, are responsible for the formation of a dislocation structure in steel TCO. The produced hardening is often accompanied by recrystallization processes, which, with the accumulation of deformation, are monotonously repeated from cycle to cycle. Moreover, recrystallization centers are formed in those parts of the lattice that are most distorted, including at the grain boundaries and their joints, which leads to the formation of a fine-grained structure. The recrystallization process that takes place during the TCO can 
be represented as repeated alternation of small strains and subsequent recrystallization annealing.

In this case, it becomes clear why in 10KHSND steel, in comparison with 08ps steel, the process of reducing the different grain size shifts toward a larger number of cycles. This is due to the fact that in alloyed steels the thermodynamic activity of carbon is lower than in carbon steels; therefore, structural changes during TCO in them occur more slowly. Thus, the influence of alloying elements on the number of cycles in the TCO will be such that with an increase in their percentage in structural steel, an increase in the number of cycles is required to obtain an equiaxed fine-grained structure.

The initial microstructure of structural steels before the TCO significantly affects the final average grain size. Naturally, the coarser initial structure of the steels (supply + annealing at $900^{\circ} \mathrm{C}$ ), in comparison with the structure in the delivery state, corresponds to the coarser-grained structure after the TCO (see Fig. 6, 8). In fig. Figure 6 shows the structure of $08 \mathrm{ps}$ steel after a five-fold TCO. Before thermocyclic treatment, the test samples in the delivery state underwent a 30-minute annealing at $900{ }^{\circ} \mathrm{C}$, due to which an equilibrium structure was formed in them. With such processing, as with the TCO of samples in the delivery state, after the first cycle, a structure with different grain sizes is formed in different areas, but the grain size in this case is slightly smaller and the grain size is larger. An increase in the number of TCO cycles leads to further grain refinement, and after the fifth cycle, a fairly uniform structure with fine grain is formed. In this case, the structure of 10KHSND steel at different stages of processing compared to the previous steel has a lower grain size, and after the fifth cycle it becomes a homogeneous fine-grained structure. Thus, with an increase in the degree of alloying of steels, a finer-grained structure is formed in them both after high-temperature annealing and in the state of factory supply.

The austenitic transformation upon heating usually begins at the intersections of carbides with high-angle boundaries of ferritic grains. In this case, the growth of austenitic grains around various carbides occurs rather quickly, and austenitic grains grow until they begin to come into contact with each other. If contact has occurred, then further growth of austenitic grains occurs with an increase in exposure time and an increase in temperature.

Thus, during thermocyclic processing, when grain growth is suppressed by low austenitization temperatures and low shutter speeds, the optimal initial structure will be such a structure that provides the maximum density of carbides at high-angle boundaries of ferrite grains. In our case, only low-alloy steel 10HSND can have this possibility.

The presence of a deformed structure in front of the TCO introduces significant changes for both 08ps carbon steel and 10KHSND low-alloy steel (see Fig. 7, 9). In this case, a finer-grained structure is formed than for samples that are in the factory-supplied state and in the state (supply + annealing at $900{ }^{\circ} \mathrm{C}$ ), which is associated with the activation of structural changes in the metal that occur during heating and cooling.

Such a significant refinement of the structure during the thermal stability process that underwent cold plastic deformation is explained by the fact that cold deformation redistributes and increases the density of imperfections in the crystal structure of dislocation stacking defects, vacancies, and also promotes the formation and development of small and high angle boundaries. Defects of the crystal lattice strongly affect the formation of the structure of alloys during phase and structural transitions, plastic deformation in front of them, and also during the passage of them, can be effectively used to create the optimal structure in steel alloys. In the TCO of pre-cold-deformed samples, the number of centers of the resulting $\gamma$ phase increases and, as a result of this, austenite grain is intensively crushed. In addition, during deformation in the intercritical temperature range as a result of the dynamic $\gamma \rightarrow \alpha$ transformation, the recrystallization process can be significantly accelerated, the constituent structures are strongly riveted and the grain is ground. Thus, the use of preliminary cold plastic deformation can be effectively used in the 
process of structural engineering of structural steels, carried out in the field of pearlite transformations.

The intensive passage of structural changes during the TCO of pre-deformed steels and the final formation of an equiaxed fine-grained structure after the third cycle is confirmed by a change in the values of the Hp parameter during cyclic annealing. In fig. $5 \mathrm{~b}$ it can be seen that the Hp values for the studied steels after the fourth and fifth cycles are almost equal, which indicates the end of the effective grinding of grain size and obtaining a finegrained structure. Thus, using the passive flux-gate method, it becomes possible to control the formation of the finest-grained structure in low-carbon and low alloy steels at the early stages of their thermocyclic treatment.

\section{Conclusions}

The studies carried out made it possible to establish the relationship between the magnetic parameter Hp and structural changes in steels during TCO. It was shown that the value of Hp depends on:

1. from the original microstructure;

2. chemical composition of steels;

3. the number of cycles of the central heating station.

It can be stated that the largest changes in the values of the magnetic parameter Hp are observed during the first heating-cooling cycles. A further increase in the number of TCO cycles decreases the magnetic amplitude Hp, which is associated with a less significant refinement of the structure in subsequent cycles. Such a pronounced character of changes in $\mathrm{Hp}$ values must be used in passive flux-gate monitoring of the formation of a fine-grained structure in low-carbon and low alloy steels during thermocyclic processing.

\section{References}

1. N.F. Nevar, R.L. Tofpenets, D. Chirun, Casti. Metal., 2 (46), 56-59 (2008)

2. A. E. Novikov, V. A. Motorin, M. I. Lamskova, M. I. Filimonov, J. Fric. Wear., 39(2), 158-163 (2018)

3. V. Alpatov, IOP Conference Series: Materials Science and Engineering 463(3), 032103 (2018).

4. A.M. Guryev, Yu.P. Kharaev, O. A. Guryev and others., Mod. Probl. Sci. Educ., 3, 65-66 (2006)

5. E. M. Savitsky, Single crystals of refractory and rare metals, 1971

6. E. M Dokl. Savitsky, AN USSR, 198 - 2, 373-376 (1971)

7. L.P. Frantsuzova, Metall. H. Treat. Met., 4, 15-17 (1984)

8. T. M. Metall. H. Treat. Met., 12, 23-27 (1978)

9. E.I. Illarionov, Therm. Proc. Met. Prod., 71-73 (1982)

10. A. A. Shmatov, Mater. Sci., № 12, 20-29 (2014)

11. V. E. Gordienko, Welded structures of industrial buildings, structures and construction machines. Assessment of the technical condition using a passive flux-gate method (2012)

12. V. E. Gordienko, Magnetic control and calculation of MK taking into account structural heterogeneity of welded joints and corrosion damage (2008) 
1. 13 V.E. Gordienko, Technical diagnosis of welded metal structures of industrial buildings, structures and construction machines (2015) 